\title{
Editorial \\ Neonatal Resuscitation: In the Public Domain or Private Decision?
}

\section{Alan D. Bedrick, MD}

As neonatologists, we are regularly called upon to counsel parents, attend deliveries, and formulate treatment plans for extremely low birth weight infants, sometimes with limited or imprecise information. Consider the pregnant woman who presents at $8-\mathrm{cm}$ cervical dilatation at an estimated 22 to 24 weeks' gestation with inaccurate dates. Although we would prefer to have the time to discuss options for resuscitation, potential hospital courses, and possible outcomes with parents, we often don't get that opportunity when there are but minutes before delivery. Importantly, there is NEVER the luxury of future certainty in planning for such a potential resuscitation. How do we answer questions like "Will my baby have brain damage?" Or the common question "What would you do if it was your baby?" Can we honestly answer that question? Is it even appropriate or moral to let our own private and personal beliefs influence those of a parent? (Does our knowledge of potential neurologic morbidities make what we might choose for ourselves different than what we might recommend to patients?)

In this issue of the Journal, Paris and Reardon ${ }^{1}$ challenge us to critically contemplate and reflect on the responsibilities of the decision-making process in the delivery room. In the case of $H C A v$ Miller, the Texas Court of Appeals upheld the right of physicians to resuscitate a 23 weeks' estimated gestational (EGA) age infant in defiance of a parental request for nonaggressive care. In that case, a Texas jury awarded $\$ 60$ million to the infant's family because “despite the parents' wishes, the neonatologist and hospital violated the instructions of the parent, and determined that the infant was viable and so instituted resuscitative measures." On appeal, the District Court of Appeals in Texas reversed the jury's verdict under the premise that until determination of a terminal condition is made, the physician did not breach the law by instituting resuscitative measures. Until the determination of a "terminally condition" is made, resuscitation can be performed over a parent's objections. In essence, parents could not refuse life-sustaining treatment.

Section of Neonatology, Department of Pediatrics, Franklin Square Hospital Center, Baltimore, $M D$.

Address correspondence and reprint requests to Alan D. Bedrick, MD, Franklin Square Hospital Center, 9000 Franklin Square Drive, Department of Pediatrics, Baltimore, MD 21237.
What are the issues this case brings to the table? What are the determining factors that compel us to pick up a $2.5-\mathrm{mm}$ endotracheal tube and place it within the airway of an extremely low birth weight infant? Is it because a 23 -week EGA infant is viable? I find the concept of viability to be very problematic in speaking with parents. With all our wondrous technology, 23-week EGA infants can indeed survive. Does it matter what percentage of them survive? If for instance, only $1 \%$ of 23 weekers survived, does that mean we counsel parents that they are viable? Does any chance of survival imply viability? What if $35 \%$ to $40 \%$ of them survive? Does viability refer to the potential/possibility/chance of survival? Current interpretation would generally say "yes." With that in mind, legal opinions of the District Court of Appeals in Texas held that physicians are within the law to independently resuscitate infants regardless of parental wishes. Conversely, could this also be interpreted that resuscitation of such infants is not mandatory? Is there ambiguity that allows alternative decision making? Are not parents instantly the legal guardians once an infant is born (and, also acting on their behalf before birth) and thus, in theory, in control of "important" medical decisions? Legal constructs and medical opinions concerning medical decision making may be more clear as a "competent" adult makes informed decisions for his or her own personal medical care through advanced directives, living wills, etc. In the labor suite, parents serve this surrogate role on behalf of their infants.

If we believe in the theory of "vitalism," infants at the limits of viability are fundamentally identical to full-term infants in their right to all supportive measures. Yet, our clinical efforts on behalf of the extremely low birth weight infant engenders considerable mortality and morbidity. Despite (and perhaps, because of) advances in technology, and pharmacology, low birth weight infants die 40 times more often than full-term infants in the first month of life. Very low birth weight infants we care for in neonatal intensive care units comprise less than 5\% of all live births, yet account for nearly $65 \%$ of neonatal deaths. Yet, how do we balance the "right to survival" against a real and possibly overwhelming likelihood of substantial neurologic morbidity and a profoundly challenged quality of life? Do we not believe that "quality of life" is an important consideration in decision making? The greatest dilemma as we reach for that endotracheal tube is that we cannot tell which infant will do well, and which infant will have a lifetime of severe and debilitating medical problems.

As physicians and other health care providers counsel families, we know that we cannot "look into the crystal ball" and really 
prognosticate outcome for that 23 weeker; yet is there more "certainty" in discussing the infant at 25 or 26 weeks' gestation? As we try to inform parents, who is ultimately responsible for health care decisions for critically ill newborns?

Should governmental or regulatory policy influence care in the delivery room? Court rulings and governmental decrees do not have a "real time" role in the delivery room. When there are but literally moments to speak with the parents of a 23 weeks' EGA baby who is about to be born, there is no time for review of governmental regulations. Our institutional ethics committees don't function in these circumstances. The ethics committee at that point time is the neonatologist and health care providers, and very worried and scared parent (s). We strive to determine "the best interests" of the infant, and act accordingly. Depending on clinical circumstances, we often don't have all the information we would like to have to make critical decisions in the delivery room. Yet, "life at all costs" is a difficult proposition to manage in the delivery room and the neonatal intensive care unit. I would challenge those health care professionals and legal theorists who believe that every infant at the grayest shadows of the limits of viability must be aggressively supported in the delivery room consider that there are outcomes which some might view as worse than mortality. Recall the infant with the stormy, complicated and near-death NICU course who now survives challenged and compromised, with serious, profoundly debilitating medical conditions; however, we all have seen infants with equally turbulent hospital stays do remarkably well. Yet a parental request to not resuscitate an infant at 23 weeks' gestation would be untenable at 26 weeks.

We play vital roles in the lives of the babies we care for, but for a brief period of time. Following discharge from the hospital, parents live day by day with the infants whose care we have been entrusted, and who live because of our decisions; we must respect their wishes, values, and beliefs, and indeed realize that, as Paris and Reardon conclude, that "treatment decisions for extremely premature newborns whose course is uncertain or ambiguous remains with those who bear responsibility for the infant - the parents." There need not be the certainty of a grim prognosis, or a "terminal condition" for a parent to choose to allow their extremely preterm infant to pass away without technologic intervention. Most importantly, we need to support parents in that decision.

\section{References}

1. Paris J, Reardon F. Bad cases make bad law: HCA vs. Miller is not a guide for resuscitation of extremely premature newborns. J Perinatol 2001;21(8):541-4. 